\title{
QUÍMICA
}

\section{PRODUCCIÓN Y CARACTERIZACIÓN DE RECUBRIMIENTOS DE BISMUTO PRODUCIDOS CON MAGNETRÓN DESBALANCEADO PULSADO}

\author{
M. F. Ortiz*, J. E. Alfonso**, J. J. Olaya*, S. E. Rodil***
}

\begin{abstract}
RESUMEN
Ortiz M. F.; J. E. Alfonso; J. J. Olaya; S. E. Rodil: Producción y caracterización de recubrimientos de bismuto producidos con magnetrón desbalanceado pulsado. Rev. Acad. Colomb. Cienc. 37 (143): 245-251, 2013. ISSN 0370-3908.

En este trabajo se presentan los resultados obtenidos en el crecimiento de recubrimientos de bismuto sobre sustratos de vidrio común, silicio y acero inoxidable 316L, usando un sistema de pulverización catódica dc pulsado con magnetrón desbalanceado y la evaluación de la microestructura, la morfología, la composición química superficial y algunas propiedades electroquímicas se hicieron en función de la frecuencia de los pulsos que genera la fuente de descarga. La microestructura de los recubrimientos fue evaluada por difracción de rayos $\mathrm{X}$ (XRD); la morfología se evaluó mediante microscopía de fuerza atómica (AFM); la composición química se determinó mediante espectroscopia de fotoelectrones emitidos por rayos X (XPS), las pruebas electroquímicas mediante ensayos de ventanas de corrosión y la adherencia entre el sustrato y el recubrimiento se evaluó mediante pruebas de rayado. El análisis DRX indica que los recubrimientos son policristalinos, los análisis de AFM determinaron que los valores de rugosidad son del orden de $25 \mathrm{~nm}$. El análisis de XPS mostró que hay energías de ligadura asociadas a $\mathrm{Bi}$ metálico y oxido de $\mathrm{Bi}$, las pruebas electroquímicas establecieron que los recubrimientos de Bi presentan un rango de potencial entre -0,315 y 1,49 mV. Finalmente, el estudio de adherencia mostró que el recubrimiento se desprende del sustrato cuando es sometido a una carga de $4.5 \mathrm{~N}$.
\end{abstract}

Palabras Clave: Recubrimientos, pulverizacion Catodica, fotoelectrones, adherencia.

\section{ABSTRACT}

In this work present the results obtained in the growth of Bi coatings onto common glass, silicon and stainless steel $316 \mathrm{~L}$ substrates through dc unbalanced magnetron sputtering and evaluation of the microstructure, morphology, chemical superficial composition and electrochemical properties was made as a function of the frequency pulse of the power supply are presented. The microstructure of the coatings was evaluated through $\mathrm{x}$-ray diffraction (XRD); the morphology was evaluated through atomic force microscopy (AFM); the superficial chemical composition was analyzed by x-ray photoelectrons spectroscopy (XPS), electrochemical test was

\footnotetext{
* Departamento de Ingeniería Mecánica y Mecatrónica, Universidad Nacional de Colombia, AA 5997 Bogotá, Colombia.

** Grupo de Ciencia de Materiales y superficies, Departamento de Física, Universidad Nacional de Colombia, AA 5997 Bogotá, Colombia.

*** Instituto de Investigaciones en Materiales, Universidad Nacional Autónoma de México
} 
evaluated by means of corrosion windows and the adherence between coating and substrate was evaluated by streaked test. The XRD analysis shows that the coatings are polycrystalline, the AFM analysis determined that the mean rugosity of Bi coatings was of the $25 \mathrm{~nm}$. The XPS analysis shows that there are binding energies associated to metallic bismuth and oxide bismuth. The electrochemical test established that the Bi coatings show a range of potential between $-0,315$ y $1,49 \mathrm{mV}$. Finally, the adherence test show that the coatings failure occurs when is subjected to a load of $4.5 \mathrm{~N}$.

Key words: Coatings, DC sputtering, Photoelectrons, adherence.

\section{Introducción}

El estudio y mejoramiento de las propiedades de los materiales, están ligados al desarrollo tecnológico, el cual debe tener en cuenta las necesidades de la sociedad y además cuidar de no tener un impacto nocivo en el medio ambiente. Teniendo en cuenta estas premisas en los últimos años se han desarrollado dispositivos con dimensiones que van desde la micra hasta el nanómetro, estos dispositivos se han integrado en equipos de producción y caracterización en todos los ámbitos de la ciencia y han dado paso al desarrollo de una tecnología que se conoce como nanotecnología.

Una de las técnicas más importantes para producir recubrimientos nanoestructurados es el sistema de pulverización catódica dc pulsadacon magnetrón desbalanceado. La cual consiste en aplicar un pulso de voltaje positivo por un corto tiempo en el blanco, lo que genera que los electrones sean atraídos hacia el blanco. Este flujo de partículas negativas descargara totalmente o parcialmente la capa aislante generada sobre la superficie del blanco en ambientes reactivos, lo que evita descargas eléctricas [1]. Recientes estudios se han enfocado en la investigación del efecto del pulso en el plasma generado en el depósito de recubrimientos en una atmósfera reactiva y no reactiva [2-4]. Se ha reportado que hay un aumento de densidad del plasma con el incremento de la frecuencia en una atmosfera no reactiva [5].También se encontró que la temperatura del electrón durante el pulso positivo se eleva comparada con la descarga en dc y el efecto aumenta con la frecuencia [6]. Es por esto, que despierta el interés en investigar lo que sucede con la resistencia a la corrosión y las propiedades ópticas de un recubrimiento metálico al variar la frecuencia de pulso en una atmósfera no reactiva.

Por otro parte, un material interesante para estudiar en forma de película delgada es el bismuto, ya que es un metal pesado no contaminante, lo que genera que el interés por investigar este material se incremente, con el fin de remplazar otros metales pesados en algunas aplicaciones, como por ejemplo en electrodos para la detección de metales pesados [7-8]. Se han usado diferentes métodos de depósito en el crecimiento de películas de bismuto a escala nanométrica, tales como: laser pulsado [9-10], evaporación térmica [11] y "flash" [12], electrodeposición [13], "sputtering" rf y d.c [14-17]. Además, se han reportado estudios del crecimiento preferencial [18], morfología y microestructura [11] controlando la temperatura del sustrato [18], espesor [11], y la resistividad eléctrica en función del espesor de la capa de bismuto [12].

En este trabajo se crecieron películas de bismuto depositadas por la técnica de pulverización de pulsado, usando un magnetrón desbalanceado, variando la frecuencia de depósito: 0 , 40 y $80 \mathrm{kHz}$, manteniendo constante la potencia en $68 \mathrm{~W}$.

\section{Desarrollo experimental}

Se crecieron recubrimientos de bismuto utilizando un equipo de pulverización con magnetrón desbalanceado (UBM) con las siguientes características: Cámara de vacío en acero inoxidable con un diámetro de $1 \mathrm{~m}$ y una altura de $80 \mathrm{~cm}$, el sistema de vacio tiene una bomba mecánica de paletas rotativas y una bomba turbomolecular con una velocidad de bombeo del orden de $10 \mathrm{~m}^{3} / \mathrm{h}$ y $1800 \mathrm{~m}^{3} / \mathrm{h}$ respectivamente. Se empleo un blanco de bismuto metálico ( $99.99 \%$ ) inmerso en una atmosfera de argón (99.999 \%). El equipo cuenta con un magnetrón desbalanceado y un sistema de refrigeración, medidores de flujo de gases y un sistema de medición presión interna. La descarga se realizo con una fuente pulsada "advanced energy" con frecuencias de $0 \mathrm{kHZ}$ hasta $80 \mathrm{kHz}$ con un incremento de $40 \mathrm{kHZ}$. El parámetro que se mantuvo constante fue la potencia de descarga la cual se fijo en $68 \mathrm{~W}$ con lo que se logro espesores de película de $400 \mathrm{~nm}$. Los parámetros de depósito se resumen en la tabla 1.

Tabla 1. Parámetros de depósito

\begin{tabular}{|l|c|}
\hline Parámetro & Valor \\
\hline Potencia & $68 \mathrm{~W}$ \\
\hline Distancia blanco-sustrato & $50 \mathrm{~mm}$ \\
\hline Presión de trabajo & $0,5 \mathrm{~Pa}$ \\
\hline Flujo de gas argón & $9 \mathrm{sccm}$ \\
\hline Frecuencia & $0,40 \mathrm{y} 80 \mathrm{KHZ}$ \\
\hline Toff & $1 \mu \mathrm{S}$ \\
\hline t-ciclo & $30 \mu \mathrm{S}$ \\
\hline Voltaje de reverse & $15 \%$ \\
\hline
\end{tabular}


Los recubrimientos fueron depositados sobre sustratos de vidrio para hacer las pruebas de venta de corrosión. También se usaron sustratos se silicio con una orientación (100). Estas muestras se limpiaron y desengrasaron con acetona e isopropanol por 5 minutos en ultrasonido por cada solución. Los sustratos de silicio fueron utilizados para hallar tasas de depósito para cada una de las frecuencias, hacer medidas de XRD, SEM, XPS y para determinar el tiempo necesario para obtener $400 \mathrm{~nm}$ de espesor en todos los recubrimientos producidos.

El espesor de los recubrimientos se determinó con un perfilometro Dektak 150. Las mediciones se realizaron en una longitud de $200 \mu \mathrm{m}$ y un $1 \mathrm{mgf}$ en el modo de crestas.

Los patrones de XRD se obtuvieron con un equipo $X$-pert ProPanalytical con línea monocromática $\mathrm{K} \alpha$ del cobre, con longitud de onda de $1.540998 \AA$, una corriente de trabajo de $40 \mathrm{~mA}$, una diferencia de potencial de $45 \mathrm{kV}$ con un intervalo de escaneo de $20^{\circ}$ a $60^{\circ}$ con un ancho de paso de $0.02^{\circ}$ en modo Bragg-Brentano. La topografía de las películas fue observada por medio de un microscopio de fuerza atómica marca JEOL modelo JSPM 4210 en modo intermitente "tapping". Posteriormente las imágenes 3D se obtuvieron con el software WSxM 4.0, procesador de imágenes de microscopía de sonda de barrido.

Para el análisis de XPS se utilizó un sistema de ultra alto vacío (UHV) de VG-Scientific Microtech Multilab ESCA2000, con un detector analizador CLAM4 MCD con una fuente de rayos $\mathrm{X}$ de $\mathrm{Al} \mathrm{K} \mathrm{K}_{\alpha}(\mathrm{h} v=1486.6 \mathrm{eV}$ ) a $22 \mathrm{~mA}$ de intensidad de haz, con ánodo polarizado a $12.5 \mathrm{kV}$. El espectro XPS se obtuvo a $55^{\circ}$ respecto de la normal a la superficie con un paso de energía constante (CAE) $\mathrm{E}_{0}=50 \mathrm{eV}$ para espectros de inspección amplia (survey) y $\mathrm{E}_{0}=20 \mathrm{eV}$ para espectros de alta resolución. La presión se mantuvo, durante la medición, en $6 \times 10^{-9}$ mbar. La posición en energía se calibró con el orbital de $\mathrm{Ag} 3 \mathrm{~d}^{5 / 2}$ en la posición de $367.3 \mathrm{eV}$, con una resolución (FWHM) de $1.10 \mathrm{eV}$ y C $1 \mathrm{~s}$ en $285.00 \mathrm{eV}$. La posición en energía se calibró con C $1 \mathrm{~s}$ en la posición de $285.00 \mathrm{eV}$. El análisis de deconvolución de espectros se hizo con el software SDPv4.1.

Las pruebas electroquímicas se realizaron mediante ensayos de ventanas de corrosión. Para ello, se usaron ensayos de polarización potenciodinámica con un potenciostato marca GAMRY referencia 600. Se empleo como electrodo de referencia el calomel y como contra electrodo una barra de grafito, se utilizo un puente salino entre el electrodo de referencia y la celda. Todas las mediciones se realizaron en una jaula de Faraday, de manera que se garantizara la anulación de efectos de los campos electromagnéticos externos. En los ensayos electroquímicos se usó una solución base (fosfato) a temperatura ambiente con un ph de 7 marca Baker y $0.9 \%$ de $\mathrm{NaCl}$. Con un potencial inicial $\left(\mathrm{E}_{\mathrm{i}}\right):-1500 \mathrm{mV}$ Vs $\mathrm{E}_{\text {ref }} \mathrm{y}$ un potencial final $\left(\mathrm{E}_{\mathrm{f}}\right): 150 \mathrm{mV} \mathrm{Vs} \mathrm{E}_{\mathrm{oc}}$, aplicando una velocidad de barrido: $50 \mathrm{mV} / \mathrm{s}$. Los ensayos se realizaron en un área expuesta a la solución de $0.25 \mathrm{~cm}^{2}$. Para evaluar la adherencia de los recubrimientos de bismuto depositadas sobre el acero $316 \mathrm{~L}$ se realizaron pruebas de rayado con el equipo micro-tribómetrico universal II, los parámetros fueron: la carga final $20 \mathrm{~N}$, la velocidad de carga fue $30 \mathrm{~N} / \mathrm{min}$, la longitud del rayado es de $8 \mathrm{~mm}$, la velocidad de recorrido fue constante $12 \mathrm{~mm} / \mathrm{min}$. La carga se incrementaba mientras la muestra se mueve. El indentador que se utilizo fue una esfera de acero con diámetro 1/16 pulgada.

\section{Resultados y Discusión}

\subsection{Caracterización estructural y electroquímica}

En la Figura 1 se observa el patrón de DRX de los recubrimientos de bismuto depositados a tres frecuencias: $0,40 \mathrm{y}$ $80 \mathrm{kHz}$. En general las películas son policristalinas y en los patrones de difracción se destacan los planos: (003), (012), (104), (015), (006), (202) y (204) ubicados en la posición $22.49,27.16,37.93,39.61,44.6,48.7$ y 56.1 , que corresponden a las señales de difracción característicos de bismuto con fase cristalográfica romboédrica (carta PDF 00-005-0519) [19]. Las intensidad delos planos indican que las películas tienen un alto grado de cristalinidad [15].

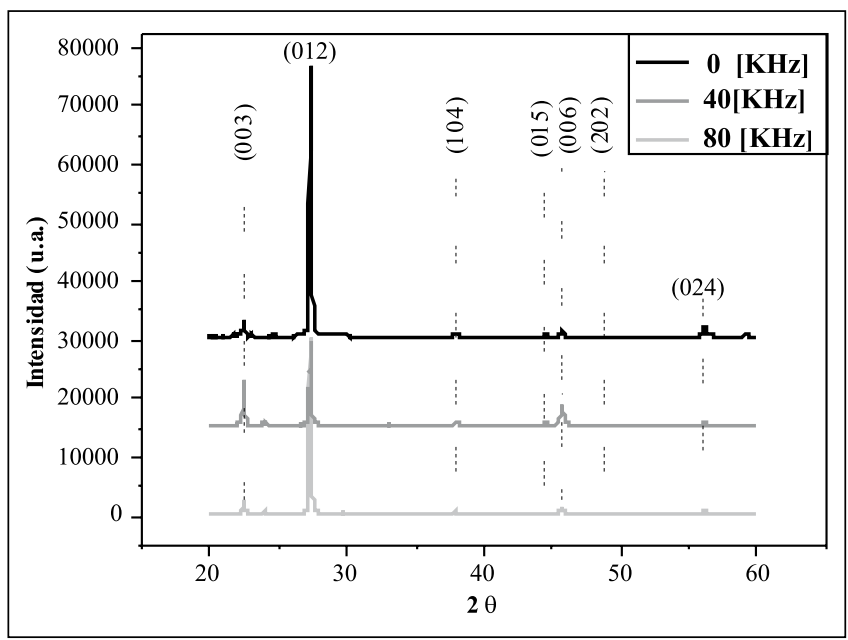

Figura 1. Patrón de difracción de rayos $\mathrm{x}$ de las películas de bismuto depositadas a diferentes frecuencias.

Las imágenes de la topografía de las películas de bismuto logradas mediante AFM se muestran en la Figura 2, donde se observa que hay un efecto de las frecuencias sobre la superficie de las películas. La imagen $2 \mathrm{~b}$ que pertenece a la 

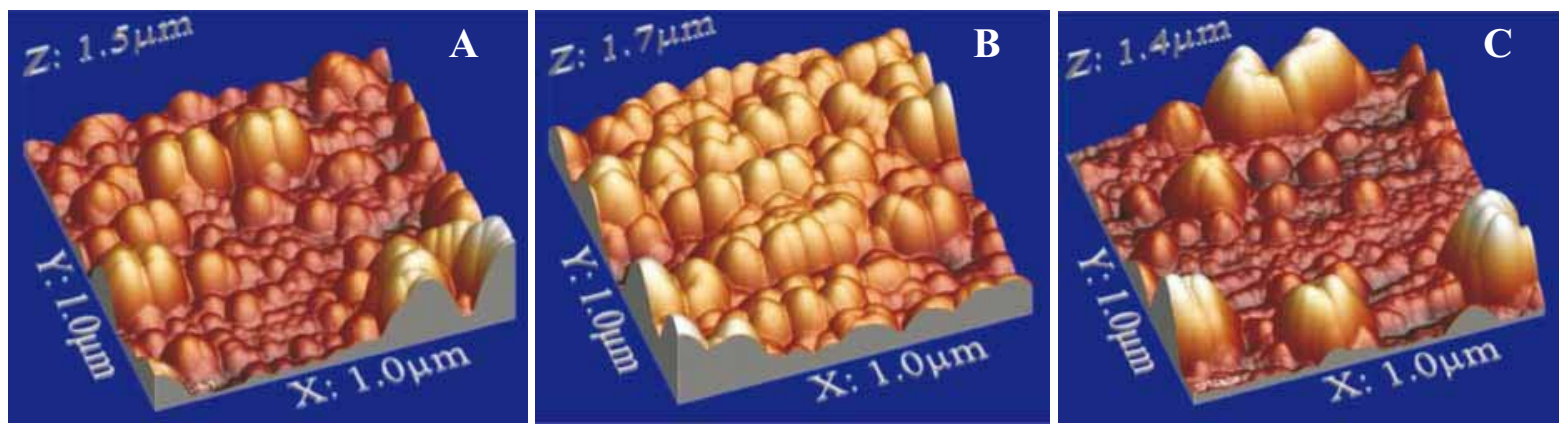

Figura 2. Imágenes de AFM de las películas de bismuto depositadas a diferentes frecuencias:

a) $0 \mathrm{kHz}$, b) $40 \mathrm{kHz}$ y c) $80 \mathrm{kHz}$

película crecida a $40 \mathrm{kHz}$ muestra una superficie con una distribución de granos más homogénea, mientras que en las otras dos películas exhiben partículas con diferentes tamaños de grano y en todos los casos más grandes que los hallados en la película crecida a $40 \mathrm{kHz}$. Esta distribución de partículas genera la alta rugosidad de las películas de bismuto las cuales son de $25.1 \mathrm{~nm}, 20.0 \mathrm{~nm}$ y $28.9 \mathrm{~nm}$ en las muestras a 040 y $80 \mathrm{khz}$, respectivamente, estos mismos resultados han sido reportados por otros autores $[9,14,20]$.

Los análisis de XPS realizados en los recubrimientos de Bi se muestran en la Figura 3, donde se aprecian los espectros de alta resolución. En el espectro se encontraron energías de ligadura de 158,77 y $164,05 \mathrm{eV}$ que corresponden al doblete $\left(4 \mathrm{f}_{7 / 2} 4 \mathrm{f}_{5 / 2}\right)$ del Bi dentro de la red cristalográfica del $\mathrm{BiO}_{2}$ y energías de ligadura de 156,785 y 162,06 eV que corresponden al mismo doblete de energía del bismuto metálico $\left(\mathrm{Bi}^{0}\right)$ [21-22]. La presencia de óxido de bismuto puede ser debido

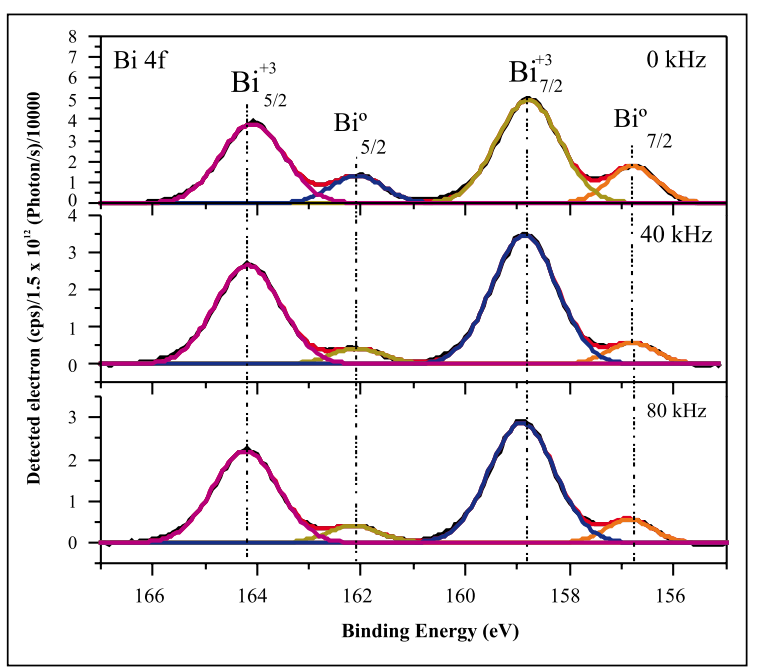

Figura 3. Resultados de de alta resolución de XPS para las películas de bismuto depositadas a las diferentes frecuencias de pulso. a una capa de pasivación que se genera en las muestras después de salir de la cámara de depósito, las energías de ligadura que se encontraron coinciden con las que se publican en la literatura [22-23].

\section{Caracterización electroquímica}

El efecto de la frecuencia de depósito sobre la ventana de potencial se presenta en la figura 4. Se observa que no hubo un cambio significativo en la amplitud de las ventas de corrosión. Para la películas depositada a $0 \mathrm{kHz}$ se obtuvo un rango de potencial desde $-0,315$ hasta $-1,47 \mathrm{mV}$, para $40 \mathrm{kHz}$ fue de $-0,315$ hasta $-1,49 \mathrm{mV}$ y para $80 \mathrm{kHz}$ fue de $-0,315$ hasta $-1,44 \mathrm{mV}$. Estos resultados permiten establecer que la reducción del ion de hidrogeno $\left(\mathrm{H}^{+}\right)$y la oxidación del bismuto (lado anódico) para los tres casos fue muy similar, lo que puede deberse a que las ventanas de potencial de trabajo dependen fuertemente de la fuerza atómica, la composición

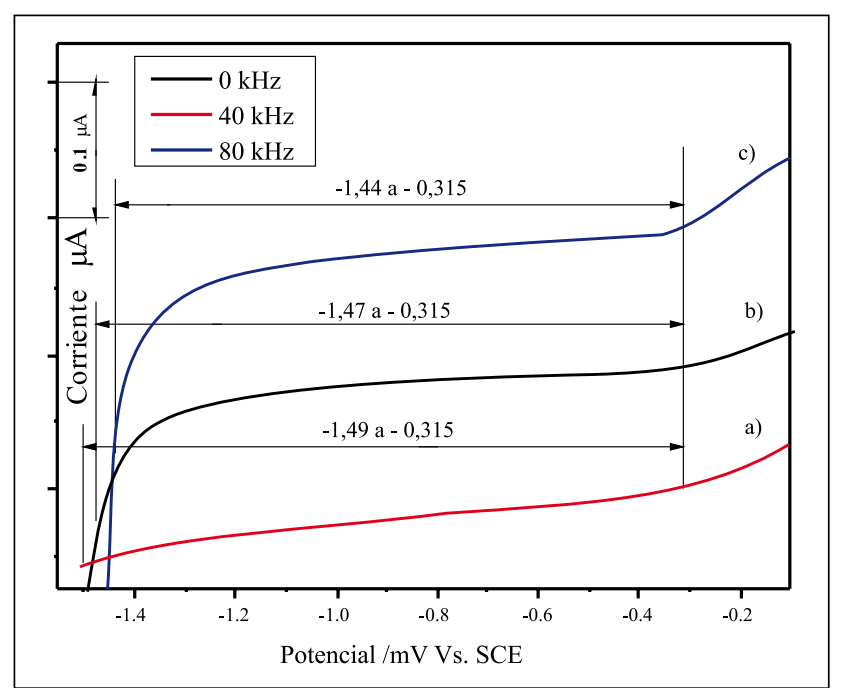

Figura 4. Ventanas de corrosión de películas de bismuto depositas: a) $0 \mathrm{kHz}$, b) $40 \mathrm{kHz} \mathrm{y} \mathrm{c)} 80 \mathrm{kHz}$ 
y en particular del PH de la solución de medición [24], como en este caso el pH fue el mismo para las tres muestra, así como también la composición, no era de esperarse un corrimiento en el en la amplitud del potencial de trabajo.

El rango o ventana de potencial presentado en esta investigación para las tres películas depositadas por pulverización dc pulsada esta en un $\mathrm{pH} 7$. Resultados similares se han encontrado en otras investigaciones [24-26], en las que se ha determinado que estos recubrimientos tienen una gran aplicación en la detección de metales pesados, por ejemplo en el agua de rio y en muestras de fertilizantes fosfatados, desplazando el uso de electrodos de mercurio en este campo, ya que el bismuto no es un metal contaminante lo que ha producido la detección de metales pesados como plomo o cadmio por métodos como voltamperometría de redisolución anódica, los picos de plomo se presentaron en potenciales de aproximadamente $-0.5 \mathrm{~V}$, mientras que el cadmio a aproximadamente $-0.7 \mathrm{~V}$ y el zinc se presento en potenciales entre $-1.0 \mathrm{y}-1.2 \mathrm{~V}$. Por lo tanto, entre más amplia sea la ventana de potencial es posible detectar mayor cantidad de elementos. En el proceso de detección se debe saber cuánto es el volumen de la muestra en la cual se está detectando los elementos.

\section{Ensayos de adherencia}

Las huellas de las pruebas de rayado sobre los tres recubrimientos se aprecian en la Figura 5. Se observa que las tres muestras presentaron el mismo comportamiento mecánico bajo una carga que se aumenta linealmente a través de la superficie recorrida y a una velocidad constante. En la prueba de rayado se identificaron cuatro principales eventos de falla, los cuales fueron etiquetados como Lc1, Lc2, Lc3 y Lc4. La descripción de las fallas correspondientes a la carga crítica se muestra en la tabla 2.

En la primera zona de la huella de rayado se presento unas fisuras del recubrimiento paralelas al movimiento del indentador, como se aprecia en la Fig. 6. en cada recubrimiento el fenómeno de fisuras en la trayectoria del indentador se generó a diferentes distancias del inicio de la huella, y por consiguiente a diferentes cargas, para la muestra depositada a 40 $\mathrm{kHz}$ se inició a $1.5 \mathrm{~mm} \mathrm{(3N)} \mathrm{(figura} 6 \mathrm{~b}$ ), para $80 \mathrm{kHz}$ se presentó casi desde el inicio de la prueba (con valores de $1 \mathrm{~N}$ ), mientras que la de $0 \mathrm{kHz}$ inició en una distancia intermedia entre 40 y $80 \mathrm{kHz}$ (con valores de 1.75N). Posteriormente, en una segunda zona se observa un rompimiento en los bordes de la huella para los tres recubrimientos, lo que indica que el recubrimiento se desprende del sustrato.

El inicio de la falla (Lc3) se presento para los recubrimientos depositados a 0,40 y $80 \mathrm{kHz}$ con las cargas $5,6 \mathrm{~N}, 6,2 \mathrm{~N}$ y $4,5 \mathrm{~N}$, respectivamente, se caracterizan por ser cargas muy bajas para aplicaciones mecánicas. Estos resultados no fueron posibles de corroborarlos con los reportados por otros autores, ya que no se encontraron investigaciones donde se realizarán pruebas de rayado sobre películas de bismuto. La baja adherencia posiblemente puede ser explicada a la diferencia del coeficiente de dilatación térmica entre el sustrato AISI 316L y el recubrimiento, que en el depósito del recubrimiento genera esfuerzos residuales de comprensión, ya que la diferencia del coeficiente de expansión térmica entre los
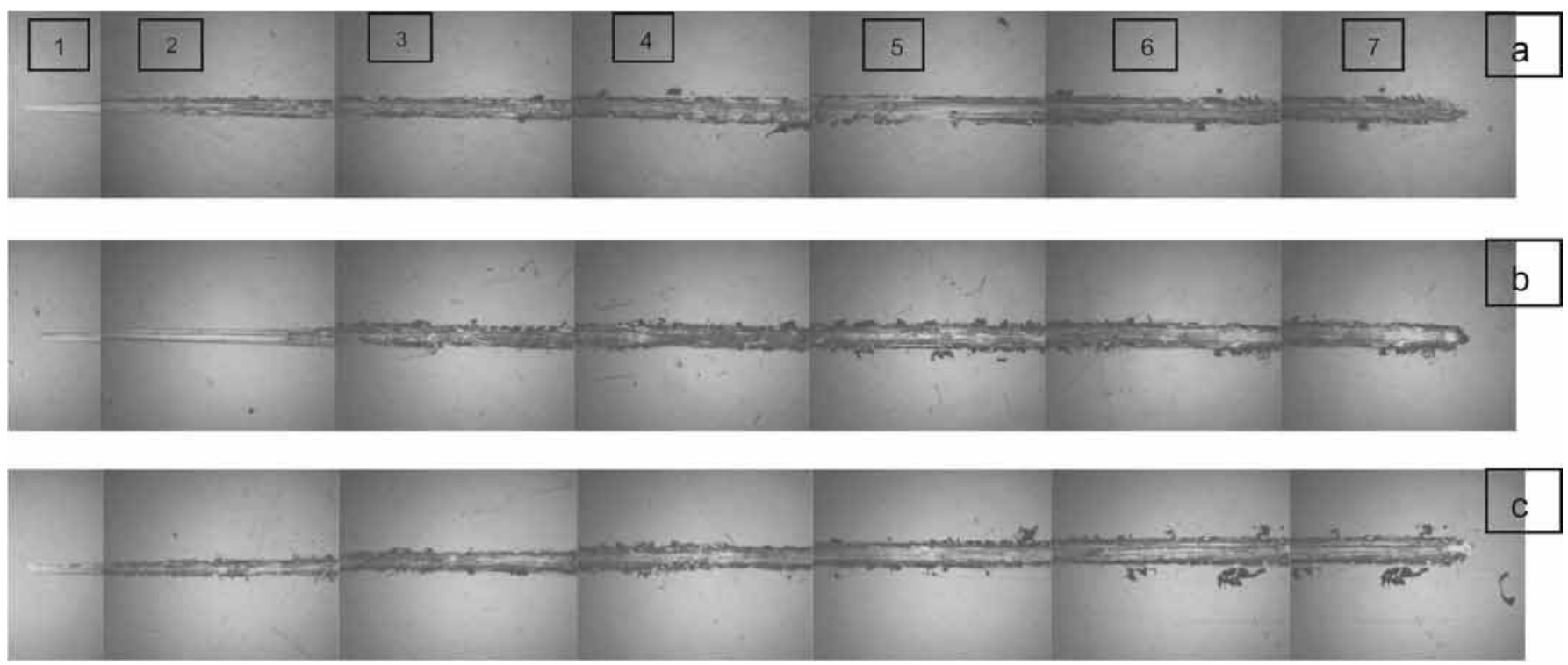

Figura 5. Huellas de las a pruebas de rayado en películas de bismuto depositadas sobre acero $316 \mathrm{~L} \mathrm{a)} 0 \mathrm{kHz}, \mathrm{b}) 40 \mathrm{kHz}$ y c) $80 \mathrm{kHz}$ 


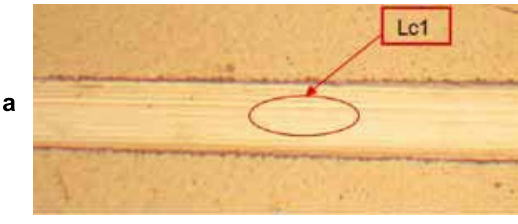

b

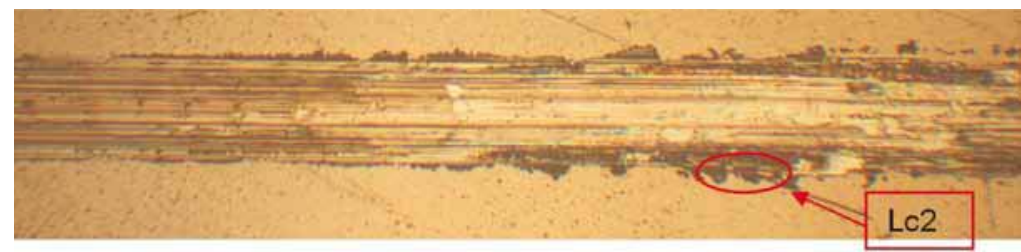

C

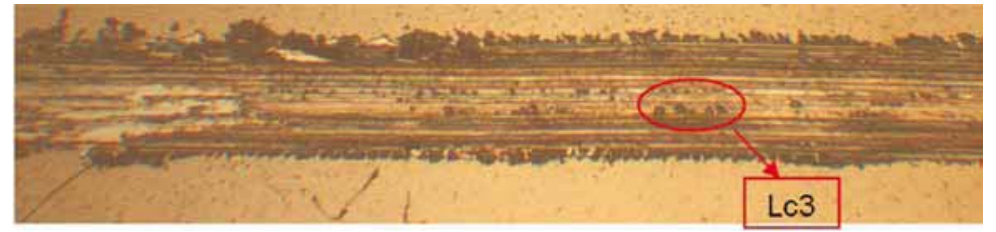

$d$

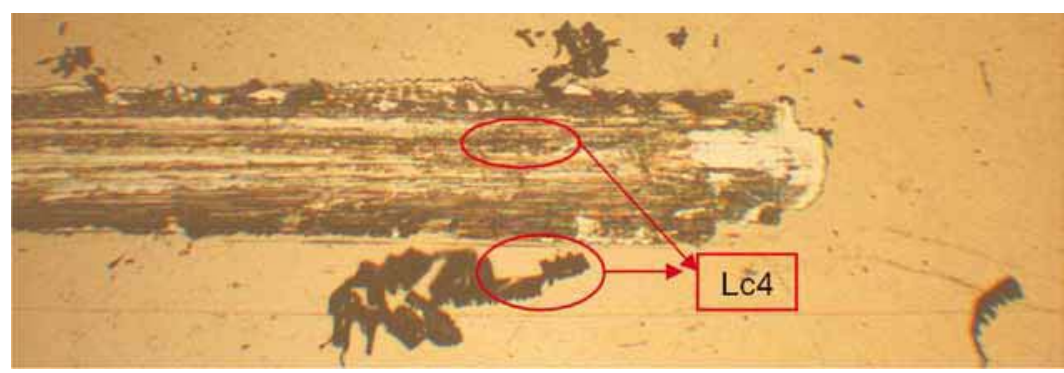

Figura 6. a) Huella total del ensayo, b )Astillamiento en los bordes de la huella, c) Falla inicial del recubrimiento d) Grietas paralelas al movimiento del indentador.

dos materiales es positiva. Otra posible explicación para al baja adherencia puede ser debido a la diferencia de parámetros de red [27], ya que para el acero inoxidable son $a=b=$ $2,9440 \AA$ y c=4,6780 $\AA$, para el bismuto los parámetros de red son $\mathrm{a}=\mathrm{b}=4,5460 \AA \mathrm{y} \mathrm{c}=11,86 \AA$.

Tabla 2. Descripción de los eventos de falla usados en la prueba de rayado

\begin{tabular}{|c|l|}
\hline Carga Crítica & Descripción de la falla \\
\hline Lc1 & Fisuras iniciales \\
\hline Lc2 & Astillado en los bordes de la pista \\
\hline Lc3 & Falla inicial del recubrimiento \\
\hline Lc4 & Falla total del recubrimiento \\
\hline
\end{tabular}

\section{Conclusiones}

Se depositaron con éxito recubrimientos de bismuto con una estructura cristalina romboédrica. El análisis AFM de los recubrimientos depositados revelo superficies con alta rugosi- dad debido a los gotas que se formaron al llegar partículas liquidas a la superficie del sustrato,donde se obtuvo que a bajas frecuencias hay una menor rugosidad ya que el tamaño de los gotas es más homogéneo que en las películas obtenidas a altas frecuencias.

Los resultados de la venta de corrosión se obtuvieronen un rango de potencial mas amplio para la película depositada a $40 \mathrm{kHz}$, siendo esta condición la que potencialmente permite que este material sea usado como electrodo en la detección de metales pesado, ya que este amplio rango permite identificar metales en potencial más negativos.

\section{Agradecimientos}

Los autores agradecen el soporte económico recibido por la dirección de investigación de la Universidad Nacional de Colombia sede Bogotá, a través del proyecto No. 203010016843 y al Proyecto Bisnano. 


\section{Referencias}

[1] L. B. Jonsson, et al., "Frequency response in pulsed DC reactive sputtering processes," Thin Solid Films, vol. 365, pp. 43-48, 2000.

[2] A. Belkind, et al., "Characterization of pulsed dc magnetron sputtering plasmas," New Journal of Physics, vol. 7, pp. 90-90, 2005.

[3] J. W. Bradley, et al., "A study of the transient plasma potential in a pulsed bi-polar dc magnetron discharge," Plasma Sources Science and Technology, vol. 13, pp. 189-198, 2004.

[4] M. J. Jung, et al., "Polycrystalline Si thin film growth on glass using pulsed d.c. magnetron sputtering," Thin Solid Films, vol. 420-421, pp. 429-432, 2002.

[5] H. Bäcker, et al., "Time-resolved investigation of plasma parameters during deposition of Ti and TiO 2 thin films," Surface and Coatings Technology, vol. 174-175, pp. 909-913, 2003.

[6] J. W. Bradley, et al., "Time-resolved Langmuir probe measurements at the substrate position in a pulsed mid-frequency DC magnetron plasma," Surface and Coatings Technology, vol. 135, pp. 221-228, 2001.

[7] A. Economou, "Bismuth-film electrodes: recent developments and potentialities for electroanalysis," TrAC Trends in Analytical Chemistry, vol. 24, pp. 334-340, 2005.

[8] H. Xu, et al., "A Nafion-coated bismuth film electrode for the determination of heavy metals in vegetable using differential pulse anodic stripping voltammetry: An alternative to mercury-based electrodes," Food Chemistry, vol. 109, pp. 834-839, 2008.

[9] M. O. Boffoué, et al., "Pulsed laser deposition of bismuth in the presence of different ambient atmospheres," Thin Solid Films, vol. 322, pp. 132-137, 1998.

[10] A. Jacquot, et al., "The effect of different scanning schemes on target and film properties in pulsed laser deposition of bismuth," Applied Surface Science, vol. 156, pp. 169-176, 2000.

[11] L. Kumari, et al., "Effects of deposition temperature and thickness on the structural properties of thermal evaporated bismuth thin films," Applied Surface Science, vol. 253, pp. 5931-5938, 2007.

[12] X. Duan, et al., "Structure and electrical properties of bismuth thin films prepared by flash evaporation method," Materials Letters, vol. 61, pp. 4341-4343, 2007.

[13] S. Jiang, et al., "Synthesis of bismuth with various morphologies by electrodeposition," Inorganic Chemistry Communications, vol. 6, pp. 781-785, 2003.

[14] D.-H. Kim, et al., "Structure and electrical transport properties of bismuth thin films prepared by RF magnetron sputtering," Applied Surface Science, vol. 252, pp. 3525-3531, 2006.
[15] S. Cao, et al., "Template-catalyst-free growth of single crystalline Bismuth nanorods by RF magnetron sputtering method," Solid State Communications, vol. 149, pp. 87-90, 2009.

[16] J. C. G. d. Sande, et al., "Optical properties of pulsed laser deposited bismuth films,” Journal of Applied Physics, 1996.

[17] J.-H. Hsu, et al., "Substrate dependence of large ordinary magnetoresistance in sputtered Bi films," Journal of Magnetism and Magnetic Materials, vol. 272-276, Part 3, pp. 1769-1771, 2004.

[18] A. Martínez, et al., "Growth and characterization of bismuth and antimony thin films," Journal of Crystal Growth, vol. 174, pp. 845-850, 1997.

[19] P.-P. JCPDS, “00-005-0519."

[20] S. Mammeri, et al., "Sputtering and surface state evolution of Bi under oblique incidence of $120 \mathrm{keV} \mathrm{Ar}+$ ions," Nuclear Instruments and Methods in Physics Research Section B: Beam Interactions with Materials and Atoms, vol. 269, pp. 909-914, 2011.

[21] T. Romann, et al., "Electroless deposition of bismuth on $\mathrm{Si}(111)$ wafer from hydrogen fluoride solutions," Thin Solid Films, vol. 518, pp. 3690-3693, 2010.

[22] G.-H. Hwang, et al., "An electrochemical sensor based on the reduction of screen-printed bismuth oxide for the determination of trace lead and cadmium," Sensors and Actuators B: Chemical, vol. 135, pp. 309-316, 2008.

[23] C. Hinnen, et al., "A comparative X-ray photoemission study of $\mathrm{Bi} 2 \mathrm{Sr} 2 \mathrm{CaCu} 2 \mathrm{O} 8+\delta$ and $\mathrm{Bi} 1.6 \mathrm{~Pb} 0.4 \mathrm{Sr} 2 \mathrm{CaCu} 2 \mathrm{O} 8+\delta^{\prime}$, , Journal of Electron Spectroscopy and Related Phenomena, vol. 73, pp. 293-304, 1995.

[24] R. Pauliukaite, et al., "Characterization and Applications of a Bismuth Bulk Electrode," Electroanalysis, vol. 16, pp. 719-723, 2004.

[25] E. A. Hutton, et al., "An introduction to bismuth film electrode for use in cathodic electrochemical detection," Electrochemistry Communications, vol. 3, pp. 707-711, 2001.

[26] C. Kokkinos, et al., "Lithographically fabricated disposable bismuthfilm electrodes for the trace determination of $\mathrm{Pb}$ (II) and $\mathrm{Cd}$ (II) by anodic stripping voltammetry," Electrochimica Acta, vol. 53, pp. 5294-5299, 2008.

[27] M. A. G. Botero, "Caracterización de las propiedades tribológicas de los recubrimientos duros," Universidad de Barcelona, Barcelona, 2005 .

Recibido: 4 de octubre de 2012

Aceptado para su publicación: 4 de junio de 2013 